\title{
ОПЫТ ИСПОЛЬЗОВАНИЯ СИСТЕМ ОЦЕНКИ И АНАЛИЗА ЭФФЕКТИВНОСТИ БИЗНЕСА В СТРАТЕГИЧЕСКОМ УПРАВЛЕНИИ
}

\author{
(c) 2021 Гавель Ольга Юрьевна
}

$\mathrm{PhD}$, к.б.н., доцент департамента бизнес-аналитики факультета налогов, аудита и бизнес-анализа Финансовый университет при Правительстве Российской Федерации, Россия, Москва

E-mail: oygavel@fa.ru

В статье рассматривается практика использования наиболее востребованных и признанных академической наукой систем оценки и анализа эффективности бизнеса, дается их сравнительная характеристика, выделяются области применения, преимущества и недостатки. При этом уточняются основные проблемы в оценке эффективности функционирования сложных экономических систем, а также основополагающие принципы построения методик оценки эффективности бизнеса. На основании проведенного исследования выделяются общие характерные компоненты систем оценки эффективности бизнеса.

Ключевые слова: эффективность бизнеса, измерение эффективности, управление эффективностью, системы оценки эффективности бизнеса.

Проблема измерения эффективности бизнеса не нова, но за последние три десятилетия к ней проявляется особый интерес и происходит ускоренное развитие в связи с тем, что системы оценки эффективности стали важными инструментами управления для реализации стратегии и контроллинга в организациях. Причем, само понимание эффективности, критерии и показатели её оценки подвергаются непрерывному изменению в соответствии с этапами общественного развития [1]. Если в условиях плановоадминистративной экономики основными критериями служили нестоимостные оценки социальной ответственности, микроэкономической сбалансированности и непрерывности бизнеса, то в условиях рыночных отношений произошло смещение фокуса в сторону стоимостных оценок деятельности бизнеса с позиций достижения интересов его собственников. В современной экономике в призму эффективности деятельности организаций включаются интересы различных групп основных стейкхолдеров, а отсутствие конфликтов между ними становится главным условием непрерывности бизнеса [2].

Трудности в оценке эффективности функционирования сложных экономических систем обусловлены:

- ограниченностью и нерациональностью распределения экономических ресурсов, что связано с непрерывностью изменений экономической среды и корректировки приоритетов развития;
- стремлением экономических субъектов к достижению высокой текущей результативности и экономичности нередко в ущерб стратегической конкурентоспособности;

- необходимостью предотвращения негативного влияния внешних факторов и потребности в непрерывной адаптации к меняющейся внешней среде ведения бизнеса.

По мнению ряда авторов, различия в определении эффективности отражают большую приверженность менеджмента организации к одному из трех общих методологических подходов: многопараметрическому, системному или целевому. При этом каждый из подходов имеет свои преимущества и недостатки [3, 4], поэтому представляется целесообразным диагностировать эффективность деятельности организации на основе синтеза наиболее сильных сторон каждого из них. При этом необходимо учитывать основополагающие принципы построения методик оценки эффективности бизнеса вне зависимости от используемых подходов:

- идентификация стратегических целей развития организации и входящих в её состав бизнес-единиц;

- формирование каскадируемой системы показателей с учетом обеспечения их сопоставимости для оценки результативности и эффективности бизнеса;

- учет стадии жизненного цикла бизнеса при выборе его приоритетных оценок;

- обоснование способа формирования ин- 
тегрального показателя для оценки уровня совокупной результативности и, соответственно, эффективности;

- определение значений и форм индикаторов, характеризующих значимость каждой бизнес-единицы.

Для оценки эффективности деятельности организации, как правило, используются многоуровневые системы, предназначенные для отражения состояния и динамики наиболее значимых для непрерывности бизнес-деятельности сфер функционирования, в которых предприятие стремится достигнуть конкретных целей. При этом создаются необходимые условия для того, чтобы производить оценку и анализировать эффективность действий менеджмента и персонала на любом организационном уровне. Внедрение и использование подобных систем относится к числу наиболее важных задач в информационно-аналитическом обеспечении адаптивного управления любой организацией.

Следует отметить, что во взглядах большинства современных авторов преобладает точка зрения на необходимость использования системного подхода к управлению эффективностью бизнеса в условиях повышенной неопределенности бизнес-среды. Однако основным принципом его реализации должно стать расширение источников информации о состоянии бизнеса организации и бизнес-среды, интеграции подсистем планирования, организации, учета, контроля и анализа на основе целеположения, базирующегося на целях управления, связанных с необходимостью информационного обеспечения различных групп стейкхолдеров бизнеса. Практический аспект действий для обеспечения эффективности бизнеса предполагает:

- обеспечение его непрерывности, т.е. способности в течении длительного периода осуществлять как минимум простое воспроизводство, выполняя при этом все взятые на себя обязательства по отношению к основным группам выгодоприобретателей;

- сохранение, а в благоприятных условиях, приумножение стоимости бизнеса для акционеров с обеспечением формирования добавленной акционерной стоимости;

- совершенствования бизнес-модели с целью своевременного выявления перспективных рыночных сегментов (потребительских и продуктовых) для обеспечения своевременного присутствия в них, и уменьшения потенциала угроз в результате реализации операционных, финансовых и иных рисков;

- совершенствования цепочек формирования стоимости, своевременной идентификации неконкурентоспособных бизнес-процессов и операций, обеспечения последующей оптимизации цепочки формирования стоимости для потребителя на основе предложения ему товара высокого качества по минимальным ценам для достижения максимальной лояльности;

- создания действенной системы мотиваций для обеспечения генерации инноваций и замены системы административного планирования и контроля на режим самоконтроля и инициации поиска и реализации потенциалов экономического роста (краткосрочный горизонт) и развития (долгосрочный горизонт).

В настоящее время разработано более двух десятков систем оценки эффективности деятельности (Performance Measurement System, PMS), получивших практическую реализацию и признанных академической наукой. К наиболее востребованным относятся модели [4-13]:

- панели управления (Tableau de Bord);

- пирамиды эффективности (Performance Pyramid);

- сбалансированной системы показателей (Balanced Scorecard, BSC) Д. Нортона и Р. Каплана;

- сбалансированной системы показателей Л. Мейселя;

- оценки эффективности деятельности и роста (Effective Progress and Performance Measurement, EP2M) К. Адамса и П. Робертса;

- внутреннего рынка Хьюлетт-Паккард;

- процессно-ориентированного анализа рентабельности (Activity-Based Performance Analysis, ABPA);

- призмы результатов деятельности (The Performance Prism System, PPS) Э. Нили, К. Адамса и М. Кеннерли.

Результаты проведенного сравнительного анализа концепций многоуровневой оценки эффективности бизнеса показаны в табл. 1. Оценка проводилась с использованием практического опыта крупных консалтинговых компаний, специализирующихся на стратегическом управлении.

На основании представленного исследования можно обозначить основные преимущества, получаемые менеджментом компании при внедрении выделенных выше систем оценки эффективности бизнеса как инструмента управле- 
Таблица 1. Результаты сравнительного анализа концепций оценки эффективности стратегического управления

\begin{tabular}{|l|c|c|c|c|c|c|c|}
\hline \\
Характеристики
\end{tabular}

ния реализацией стратегии:

- обеспечивается необходимая направленность и последовательность действий, соответствующая выработанной стратегии на всех организационных уровнях управления;

- формируются предпосылки и возможности принятия рациональных решений относительно распределения ресурсов;

- углубляется на качественном уровне проработка стратегических ориентиров развития компании и структурируется процесс управления её осуществления;

- формируются возможности управления развитием компании с учетом потенциала создания стоимости за счет нематериальных и иных неидентифицируемых на балансе компании активов;

- достигается большая степень прозрачности бизнеса и создаются предпосылки для организации эффективной системы внутреннего контроля и риск-менеджмента;

- предоставляется возможность оперативно актуализировать стратегию развития организации.

С точки зрения достижения задачи диагностики стратегической эффективности развития компаний среди многоуровневых систем оценки, в наибольшей степени отвечающим современным вызовам, являются BSC Д.Нортона и Р.Каплана, модель ЕР2М, метод АВРА М.Мейеpa, призма PPS Э. Нили, К. Адамса и М. Кеннерли.
Среди моделей, наиболее востребованных как в российском бизнесе, так и во всем мире, в порядке их приоритетности выделяются: сбалансированная система показателей Д.Нортона и Р.Каплана, система процессно-ориентированного управления рентабельностью АВРА, призмы результатов деятельности PPS. Другие модели, как правило, пользуются региональными предпочтениями.

Идея концепции призмы результатов деятельности PPS заключается в учете потребностей ключевых стейкхолдеров, что позволяет бизнесу устойчиво развиваться и поддерживать интересы его акционеров в долгосрочной перспективе [12]. Несмотря на то, что существуют системы сбалансированных показателей (финансовых и нефинансовых), имеется настоятельная потребность в моделях, помогающих организациям обновлять и (или) разрабатывать карты показателей, соответствующие требованиям конкурентной среды, где интересы ключевых заинтересованных сторон ставятся на первое место. Основываясь на стратегии измерения отношений, система PPS может быть представлена геометрической призмой, содержащей пять взаимосвязанных аспектов оценки:

- удовлетворенность стейкхолдеров (идентификация ключевых заинтересованных сторон, их требований и уровня удовлетворенности);

- стратегия (определение стратегий, которым компания должна следовать для удовлет- 
ворения потребностей ключевых стейкхолдеров бизнеса);

- процессы (идентификация бизнеспроцессов, необходимы для реализации этих стратегий, и возможностей их улучшения);

- возможности (определение возможностей и ресурсов, необходимых для управления этими процессами и их улучшением);

- вклад заинтересованных сторон (вклад, который компания хочет иметь от заинтересованных сторон для поддержания и развития этих возможностей).

Авторы считают, что ответы на данные вопросы позволят сформировать систему показателей в рамках каждой из перспектив, которая затем используется для каскадирования по организационным уровням компании. Индикаторы, определяющие цели организации, ее стратегии, процессы и возможности в PPS интегрируются в картах успеха.

Таким образом, отличительной особенностью данной системы является: во-первых, проведение стейкхолдеровского анализа перед формированием стратегии организации; во-вторых, идентификация процессов и возможностей, требуемых для поддержки этой стратегии, перед созданием соответствующих показателей эффективности. Измеряемыми параметрами процессов / подпроцессов / их компонентов являются количество, качество, время, простота использования, стоимость, инновационность (персонала и технологий).

Ряд экспертов считают, что призма эффективности представляет собой инструмент наиболее целесообразный к применению для организаций государственного и третьего сектора экономики (особенно для малого и среднего бизнеса), а также в компаниях, функционирующих в регулируемых отраслях или на базе государственно-частного партнерства [14, 15]. Слабыми сторонами системы можно назвать ее непригодность для целей бенчмаркинга и недостаточно четкое описание процедур выбора показателей результативности деятельности. Также следует отметить, что применение данной модели связано с внедрением процессного подхода в управлении. По мнению российского менеджмента, это сопряжено с множеством операционных трудностей, поскольку требует у большинства компаний модификации своих бизнес-процессов, перехода от вертикальных структур к горизонтальным. Однако, по сравне- нию с другими системами оценки эффективности, PPS имеет ряд следующих основных преимуществ:

- фокусируется на всех ключевых группах стейкхолдеров;

- не игнорирует согласование стратегии, процессов и возможностей с интересами заинтересованных сторон;

- не только учитывает ожидания стейкхолдеров, но и делает акцент на обратную связь, т.е. рассматривает их вклад в устойчивое функционирование самой компании.

Система процессно-ориентированного анализа рентабельности АВРА может стать значимым инструментом измерения и повышения инвестиционной привлекательности организации, связывая воедино осуществляемые в интересах клиентов бизнес-процессы, их затраты и доходы, что позволяет оценить рентабельности отдельных бизнес-процессов, трансакций или продуктов. Такая взаимосвязь делает возможным проведение анализа как отдельных клиентских сегментов, так и клиентской базы в целом. В свою очередь, рентабельность работы с клиентами - фактор, определяющий рентабельность всех продуктов и трансакций. Таким образом, данная модель позволяет оценить эффективность каждого процесса и всей организации.

ABPA аналогично системе процессноориентированного учета затрат (ABC-costing) анализирует стоимость процессов и транзакций, однако с большим акцентом на факторы доходов, чем затрат. Она открывает ряд аналитических возможностей, способствующих росту эффективности деятельности компании и её стоимости, достижение которых затруднительно без применения системы АВРА:

- идентификация относительно недорогих транзакций для пакетирования их в единый продукт, чтобы реализовать его с премиальной рентабельностью;

- выявление убыточных клиентов и определение взаимодействий с ними;

- определение взаимоотношений с наиболее рентабельными клиентами на долгосрочной перспективе.

Следовательно, основными преимуществами данной системы будут возможности:

- идентификации рентабельности процессов;

- увязки того или иного процесса с конкретным потребительским сегментом; 
- анализа эффективности по всем уровням организации как сверху вниз, так и снизу вверх.

- использования достаточно простого для определения показателя рентабельности клиентов, что позволяет руководителям подразделений на его основе самостоятельно формировать стратегию взаимоотношений с потребителями.

Корректное применение системы АВРА требует учета ситуаций при возникновении различий в ассортименте и (или) ценах на продукцию в различных подразделениях, а также контроля стратегий основных и вспомогательных подразделений на предмет их непротиворечивости. Идеальной будет организация производства, при которой каждый бизнес-процесс имеет одного владельца.

Однако сложность системы АВРА обуславливает ограничения ее практического применения:

- взаимосвязь с гибкой системой АВСcosting, изменяющейся при модернизации организационных принципов и технологии [16];

- трудность анализа клиентских транзакций в режиме реального времени;

- оценка эффективности подразделений по выполняемым ими транзакциям, связанная с проблемой распределения удельного дохода от клиента по транзакциям, что является одним из самых «узких мест» в применении системы, т.е. она в большей мере соответствует задачам управления «плоскими» бизнес-структурами, ориентированными на клиента.

- трудности учета и оценки факторов, определяющих нефинансовые результаты в разрезе отдельных клиентов;

- сложность оценки влияния на доходы действий персонала, выполняемых в интересах всех клиентов;
- необходимость процессного учета и сложность внедрения;

- отсутствие методологического аппарата для определения интегрального показателя, используемого для оценки эффективности бизнеca.

В настоящий момент система сбалансированных показателей Д.Нортона и Р. Каплана является наиболее востребованной во всем мире, предоставляя топ-менеджерам и собственникам возможность перевести стратегические цели компании на уровень операционной деятельности. Она позволяет четко понять, как отдельные подразделения, проекты компании, отдельные сотрудники влияют на достижение поставленных перед компанией целей, дает возможность корректировать их деятельность, направляя ее на выполнение стратегии. Цели и показатели системы формируются в зависимости от видения и стратегии организации, при этом деятельность организации рассматривалась авторами в разрезе четырех проекций оценки: финансы, клиенты, процессы, персонал и развитие [5]. Типичные драйверы создания стоимости компании в модели BSC представлены в табл. 2.

Наполнение проекций показателями и обоснование их целевых, допустимых и критических значений возможно только после установления стратегических целей бизнес-системы и определения ключевых факторов успеха их достижения по уровням организации бизнеса (от топ-менеджмента до рабочего места). Построение системы осуществляется таким образом, чтобы задачи и индикаторы более высокого уровня в интегрированном виде отражали задачи и показатели более низкого уровня. Однако глубина внедрения и соблюдения авторских технологий на практике в каждом индивидуаль-

Таблица 2. Типичные драйверы создания стоимости компании в модели BSC

\begin{tabular}{|l|l|}
\hline \multicolumn{1}{|c|}{ Проекции } & \multicolumn{1}{|c|}{ Характеристика ключевых показателей } \\
\hline Финансы & $\begin{array}{l}\text { Состояние и динамика отношений с инвесторами, регуляторами финансового рынка, } \\
\text { драйверы создания стоимости: рост рентабельности продаж, рентабельности собствен- } \\
\text { ного капитала, инвестированного капитала, свободного денежного поток, EVA, MVA и пр. }\end{array}$ \\
\hline Клиенты & $\begin{array}{l}\text { Состояние и динамика развития отношений с клиентами, драйверы формирования ло- } \\
\text { яльности клиентов и роста их маржинальности: доля рынка, сохранение и расширение } \\
\text { клиентской базы, удовлетворение запросов, прибыльность и пр. }\end{array}$ \\
\hline $\begin{array}{l}\text { Внутренние } \\
\text { бизнес-процессы }\end{array}$ & $\begin{array}{l}\text { Состояние и динамика развития процессов и их инновационности, драйверы создания } \\
\text { конкурентных преимуществ: улучшение обслуживания клиентов, повышение эконо- } \\
\text { мической, экологической, организационно-технологической эффективности и пр., в } \\
\text { значительной степени определяемые спецификой бизнеса }\end{array}$ \\
\hline $\begin{array}{l}\text { Персонал } \\
\text { и развитие }\end{array}$ & $\begin{array}{l}\text { Состояние и динамика развития персонала, драйверы создания лояльности персонала и } \\
\text { ключевых компетенций: индекс удовлетворенности сотрудников, их лояльность, квали- } \\
\text { фикация, генерация инициатив, эффективность работы информационной системы и пр. }\end{array}$ \\
\hline
\end{tabular}


ном случае значительно варьирует, зачастую используется только инструментарий системы или происходит её частичное внедрение [27].

Аналитическая модель BSC для отдельно взятой компании включает несколько необходимых элементов:

- карту стратегических задач, логически связанных со стратегическими целями;

- карту сбалансированных показателей описание логики организации бизнеса с использованием параметров по нескольким перспектива, которая переводит видение и стратегию в четкие количественные цели и измеримые показатели на каждом уровне организации управления, отражая их целевые и критические значения, а также сроки достижения целевых результатов;

- целевые проекты (инвестиции, обучение и т.п.), обеспечивающие внедрение необходимых изменений;

- панели управления (Performance dashboard) для рабочих мест, занятых в бизнес-процессах, с визуализацией отклонений контролируемых показателей от планово-бюджетных с возможностью моделирования для каждого из менеджеров связей в системе «фактор - результат».

Таким образом, BSC выполняет три основные функции в организации: система стратегического управления, система измерения и инструмент коммуникации, ориентируя менеджмент на адекватные действия, преследующие цель стратегического развития и роста бизнеса, повышение его инвестиционной привлекательности и рыночной стоимости. Тем не менее, она обладает рядом недостатков, при этом чаще всего, выделяемыми менеджментом различных уровней при проведении опросов, являются [17]:

- трудность определения показателей, динамику которых можно напрямую использовать при оценке изменения стоимости компании;

- изначально не предполагает формирование единого интегрального показателя для отдельных проекций, постановка же в качестве стратегической цели роста рыночной стоимости компании может привести к исключению значимых показателей из системы оценки;

- не подразумевает улучшение уже существующих в организациях систем оценки эффективности: обычно менеджеры редко приветствуют построение система оценки эффективности с нуля;

- недостаточное внимание к фактической разработке и внедрению: рекомендации, данные авторами в отношении выбора показателей, носят скорее общий, чем конкретный характер;

- задача проектирования достаточно сложна, однако реализация этих мер представляет собой еще более сложную задачу.

Так, С.Танген, высоко оценивая новаторскую модель BSC с точки зрения создания академических и философских основ формирования системы оценки эффективности бизнеса, предоставляющей компаниям руководящие принципы для разработки их уникальной системы, одновременно с этим критикует поверхностный подход авторов к объяснению практических шагов, которые должны быть предприняты на операционном уровне [18]. Г.Носи критиковала BSC за отсутствие в ряде случаев объективности при формировании показателей эффективности и не достаточную своевременность в предоставлении информации [19].

Следует также добавить, что изначально система показателей планировалась как универсальная информационно-аналитическая модель при условии заданных ключевых целей компании. Однако именно идентификация перспектив при построении BSC в организации позволяет выделить те ключевые факторы создания стоимости компании, которые необходимо включать в основу модели. Таким образом, модель не является универсальной для всех компаний и требует учета особенности их бизнес-модели, стадии жизненного цикла, состояния ключевых компетенций и других важных условий ведения бизнеса. Обобщая информацию о практике использования системы BSC, были выявлены её основные преимущества и недостатки, представленные в таблице 3.

BSC не является статичной, представляя собой инструмент, развиваемый как его авторами, так и их последователями, с целью создания новых практических инструментов и методик, позволяющих интегрировать её в систему управления компаний [17].

Если организация функционирует в стабильной экономической среде, эффективность деятельности слабо зависит от внешних факторов, её бизнес-процессы просты и не предполагают значительных корректировок, то вполне правомерным может быть применение для оценки эффективности бизнеса традиционной финансовой модели, обладающей определенными преимуществами: простота при внедрении и 
Таблица 3. Основные преимущества и недостатки сбалансированной системы показателей

\begin{tabular}{|c|c|}
\hline & $\begin{array}{l}\text { - концентрация на стратегических бизнес-целях; } \\
\text { - использование причинно-следственных связей как инструмента управления; } \\
\text { - каскадирование целей и показателей в рамках организационной структуры компании; } \\
\text { - сбалансированность финансовых и нефинансовых показателей; } \\
\text { - увязка стратегических, тактических и оперативных задач; } \\
\text { - охват всех существенных сторон деятельности организации; } \\
\text { - ориентация на наличие обратной связи между уровнями управления; } \\
\text { - осуществление последовательного мониторинга реализации стратегии; } \\
\text { - многоаспектность и иерархичность прохождения коммуникационного процесса; } \\
\text { - возможность ориентированной на обучение коммуникации по всем уровням организации; } \\
\text { - возможность использования для мотивации; } \\
\text { - хорошая интеграция с системой контроллинга и увязка с методами управления. }\end{array}$ \\
\hline & $\begin{array}{l}\text { - не выражает интересов всех ключевых стейкхолдеров бизнеса; } \\
\text { - отсутствие простоты и ясности в методологии построения; } \\
\text { - требует значительной финансовой и компетентной поддержки со стороны собственников и менед- } \\
\text { жмента всех уровней организации; } \\
\text { - трудности с адаптацией к динамичной среде, что требует периодического пересмотра показателей } \\
\text { эффективности; } \\
\text { - возможность разработки слишком избыточного / ограниченного набора показателей, недостижи- } \\
\text { мых показателей; } \\
\text { - невозможность использования для бенчмаркинга; } \\
\text { - в случае нацеленности на повышение стоимости организации чрезмерный фокус на управлении, } \\
\text { базирующемся на показателях, игнорирующих “мягкие” факторы; } \\
\text { - не всегда обеспечивается однозначность причинно-следственных связей «цель - средство - страте- } \\
\text { гическая карта»; } \\
\text { - может тормозить мотивацию осуществления проекта; } \\
\text { - не предусмотрены механизмы разрешения конфликтов; } \\
\text { - не дает механизма определения временного горизонта достижения целей; } \\
\text { - получаемая информация может быть искажена в силу ограничений и условностей в процессе про- } \\
\text { гнозирования; } \\
\text { - внедрение системы должно проводиться параллельно с дополнительными изменениями в системах } \\
\text { планирования и мотивации персонала; } \\
\text { - более подходит для вертикально-структурированных компаний. }\end{array}$ \\
\hline
\end{tabular}

использовании, в том числе, в системе материального стимулирования.

Если структура организации характеризуется небольшим количеством вспомогательных бизнес-процессов (часто, сфера услуг) и присутствует существенная зависимость от факторов внешней среды, лучшими системами оценки эффективности можно назвать ABPA или PPS. Для компаний государственного сектора экономики, функционирующих в регулируемых отраслях или на базе государственно-частного партнерства, хорошо зарекомендовавшей себя является модель PPS.

В современных условиях организации сталкиваются с заметным влиянием на эффективность их деятельности множества факторов турбулентной внешней среды, повышением роли интеллектуального капитала, необходимостью в перманентной корректировке бизнес-процессов компании для поддержания её конкурентоспособности, адаптации бизнес-модели к складывающимся условиям ведения бизнеса, что ставит на первый план применение усовершенствованных систем BSC и PPS (особенно для среднего и малого бизнеса).

Таким образом, несмотря на определенные методологические различия в современных подходах к оценке эффективности бизнеса, можно выделить характеристики, по которым существует консенсус среди их авторов и исследователей. Измерение эффективности характеризуется как система, основанная на:

- приоритетном значении стратегических ориентиров;

- многомерных показателях эффективности (нефинансовых индикаторов, сбалансированных с финансовыми), построенных на основе стратегии организации с целью её реализации, а в некоторых случаях, и корректировки;

- причинно-следственных связях между стратегическими целями, процессами и действиями;

- обеспечении обратной связи и коммуникации, помощи в создании среды обучения;

- постоянном совершенствовании организации, заключающемся в непрерывном мониторинге развития и изменений во внешней и внутренней среде ведения бизнеса и способностью 
к их систематической взаимоувязке с самой системой.

Системы оценки эффективности характеризуются наличием следующих основных компонентов, которые предполагают выполнение их основных функций (сбор данных и информационно-аналитическое обеспечение принятия управленческих решений):

- формирование системы индикаторов для оценки эффективности, включающей процедуры идентификации потребностей стейкхолдеров, планирования, определения стратегических целей, разработки индикаторов, выбора и постановки целей;

- сбор и обработка данных, состоящие из процедур сбора и анализа данных;

- управление информацией, охватывающее процессы предоставления информации, интерпретации и принятия решений;

- оценка эффективности и мотивация, включающая процессы оценки эффективности и увязки ее с системой вознаграждения (стимулирования) персонала;

- системный обзор, включающей различные процедуры проверки, обеспечивающей наличие цикла обратной связи внутри системы

Все эти процессы могут происходить на различных уровнях управления организации.

Обзор работ по использованию систем оценки и анализа эффективности бизнеса показал, что, несмотря на большой интерес к данной об- ласти исследований, подтверждающийся многочисленностью публикаций, эмпирических исследований до сих пор недостаточно $[4-15,18,19]$. Большинство работ сосредоточены на разработке подходов к построению систем оценки или качественном анализе теоретических аспектов различных проблем оценки эффективности бизнеса. Существует большая потребность в количественных исследованиях, направленных на проверку связей между сбалансированной системой измерения эффективности и самой эффективностью бизнеса. Кроме того, в многомерных системах оценки большое внимание уделяется стратегии и ее реализации с точки зрения стратегических менеджеров, оперативный уровень и уровень рабочих мест зачастую рассматривается вскользь, несмотря на то, что их оптимальное функционирование позволяет компании достигать её долгосрочных целей и создавать конкурентные преимущества. Также следует отметить, что в большинстве работ основное внимание уделялось непосредственно самим индикаторам эффективности, представляющим только часть системы оценки. Рассмотрению других её областей, таких как сбор данных и их обработка, посвящается значительно меньшее внимание. Обозначение данных проблем ставит ориентиры, позволяющие углубиться в области дальнейшего исследования оценки эффективности бизнеса.

\section{Библиографический список}

1. Гавель О. Ю. Генезис взглядов на категорию эффективность бизнеса и особенности её оценки. // Экономические науки. 2021. № 9 (202). с. 205-213.

2. Бариленко В.И., Бердников В.В, Гавель О.Ю., Керимова Ч.В. Аналитическое обоснование конкурентоспособных бизнес-моделей: учебное пособие. / колл. авторов. - Москва: Русайнс, 2015. - 315 с.

3. Гавель О.Ю., Керимова Ч.В., Музалев С.А. Развитие методологии стратегического анализа эффективности бизнеса: монография. / О. Ю. Гавель, Ч. В. Керимова, С. А. Музалев. - Москва: КноРус, 2021. - 170 с.

4. Еремина Г. А. Системы оценки эффективности деятельности: принципы создания и применения, освещение в научной литературе. // Интернет-журнал «Науковедение». 2017. T. 9, № 6. URL: [https://naukovedenie. ru publishing@naukovedenie.ru].

5. Каплан Р.С., Нортон Д. П. Стратегическое единство: создание синергии организации с помощью сбалансированной системы показателей. / М.: Вильямс, 2006. - 384 с.

6. Майер М. Оценка эффективности бизнеса. / М.: Вершина. 2004. - 272 с.

7. Adams C, Roberts P. You are what you measure // Manufacturing Europe. -1993.

8. Franco-Santos M., Kennerley M., Micheli P., Martinez V., Mason S., Marr B., Gray D., Neely A. Towards a Definition of a Business Performance Measurement System // International Journal of Operations \& Production Management. 2007. № 27(8).

9. Khan K., Shah A. Understanding performance measurement, through the Literature // African Journal of Business Management. 2011.v. 5, № 35. pp. 13410-13418.

10. Maisel L. S. Performance Measurement. the Balanced Scorecard Approach. // Journal of Cost Management. 1992. № 6 (2). pp. 47-52. 
11. McNair C.J., LunchR.L., CrossK.F. Do financial and nonfinancial performance measures have to agree? // Management Accounting.1990. No. 11.pp. 28-36.

12. O'Neely A., Adams C., Crowe P. The performance prism in practice // Measuring Business Excellence. - 2001. - Vol. 5, № 2. - pp. 6-13.

13. Striteska M., Spickova M. Review and Comparison of Performance Measurement Systems.// Journal of Organizational Management Studies. Vol. 2012 (2012), Article ID 114900 URL: [http://www.ibimapublishing.com/journals/JOMS/ joms.html].

14. Severgnini E., Galdamez E., Moraes R. Satisfaction and contribution of stakeholders model from Performance Prism. // Brazilian Business Review. 2017. 15(2). pp. 120-134.

15. Smulowitz S. Evidence for the performance prism in higher education. // Measuring Business Excellence. 2015. 19(1). pp.70-80.

16. Акимов А. Анализ себестоимости каждого товара по методике АВС. // Консультант. 2005. № 23. С. 36.

17. Гавель О.Ю. Перспективы использования контроллинговых систем в стратегическом управлении. // Финансовая аналитика: проблемы и решения. 2017. Т. 10. № 1 (331). с. 4-19.

18. Tangen $S$. Performance measurement: from philosophy to practice. // International Journal of Productivity and Performance Management. 2004. 53(8). pp. 726-737.

19. Noci G. Accounting and non-accounting measures of quality-based performances in small firms. // International Journal of Operations and Production Management. 1995. № 15(7). pp. 78-106. 